\title{
Endoplasmic Reticulum Stress Impaired Uncoupling Protein 1 Expression via the Suppression of Peroxisome Proliferator-Activated Receptor $\gamma$ Binding Activity in Mice Beige Adipocytes
}

\author{
Ana Yuliana ${ }^{1}$, Asumi Daijo ${ }^{1}$, Huei-Fen Jheng ${ }^{1}$, Jungin Kwon ${ }^{1}$, Wataru Nomura ${ }^{1,2}$, \\ Haruya Takahashi ${ }^{1}$, Takeshi Ara ${ }^{1}$ (D), Teruo Kawada ${ }^{1,2}$ and Tsuyoshi Goto ${ }^{1,2, *}$ \\ 1 Laboratory of Molecular Function of Food, Division of Food Science and Biotechnology, Graduate School of \\ Agriculture, Kyoto University, Gokasho, Uji, Kyoto 611-0011, Japan; anayulia@kais.kyoto-u.ac.jp (A.Y.); \\ asumi.7.9.2.8@gmail.com (A.D.); ydnas@kais.kyoto-u.ac.jp (H.-F.J.); kwon.jungin.34r@st.kyoto-u.ac.jp (J.K.); \\ nom2@kais.kyoto-u.ac.jp (W.N.); haruya@kais.kyoto-u.ac.jp (H.T.); ara@kais.kyoto-u.ac.jp (T.A.); \\ fat@kais.kyoto-u.ac.jp (T.K.) \\ 2 Research Unit for Physiological Chemistry, the Center for the Promotion of Interdisciplinary Education and \\ Research, Kyoto University, Kyoto 606-8501, Japan \\ * Correspondence: tgoto@kais.kyoto-u.ac.jp; Tel.: +81-774-38-3753
}

Received: 29 December 2018; Accepted: 8 January 2019; Published: 11 January 2019

\begin{abstract}
Endoplasmic reticulum (ER) homeostasis is critical in maintaining metabolic regulation. Once it is disrupted due to accumulated unfolded proteins, ER homeostasis is restored via activation of the unfolded protein response (UPR); hence, the UPR affects diverse physiological processes. However, how ER stress influences adipocyte functions is not well known. In this study, we investigated the effect of ER stress in thermogenic capacity of mice beige adipocytes. Here, we show that the expression of uncoupling protein 1 (Ucp1) involved in thermoregulation is severely suppressed under ER stress conditions (afflicted by tunicamycin) in inguinal white adipose tissue (IWAT) both in vitro and in vivo. Further investigation showed that extracellular signal-regulated kinase (ERK) and c-Jun N-terminal kinase (JNK) were both activated after ER stress stimulation and regulated the mRNA levels of $U c p 1$ and peroxisome proliferator-activated receptor $\gamma($ Ppar $\gamma)$, which is known as a $U c p 1$ transcriptional activator, in vitro and ex vivo. We also found that Ppar $\gamma$ protein was significantly degraded, reducing its recruitment to the $U_{c p} 1$ enhancer, thereby downregulating Ucp1 expression. Additionally, only JNK inhibition, but not ERK, rescued the Ppar $\gamma$ protein. These findings provide novel insights into the regulatory effect of ER stress on Ucp1 expression via Ppary suppression in beige adipocytes.
\end{abstract}

Keywords: beige adipocytes; endoplasmic reticulum stress; peroxisome proliferator-activated receptor $\gamma$; uncoupling protein 1

\section{Introduction}

Adipose tissue has been broadly characterized as white and brown adipocytes through their distinct characteristics in lipid metabolism. White adipose tissue (WAT) functions as energy storage, while brown adipose tissue (BAT) dissipates energy as heat supported by its high oxidative capacity [1-3]. Recent findings show inducible brown-like white adipocytes, known as beige adipocytes. Beige adipocytes are developed within WAT in response to $\beta$-adrenergic receptor $(\beta-A R)$ stimulation, termed as browning of WAT, and exhibit similar characteristic as BAT $[2,4]$. The transcription of uncoupling protein 1 (Ucp1) is tightly regulated during browning, thus it is 
often used as a browning marker of adipose tissue [1,5]. Interestingly, in addition to browning, beige adipocytes also show unique plasticity to undergo whitening (reversal of browning) [6-8].

The endoplasmic reticulum (ER) is a critical organelle in sensing and handling cellular nutrient required for normal cellular functions and survival [9]. Certain physiological and pathological factors such as nutrient deprivation, lipids, or increased synthesis of secretory proteins can induce cell stress and disrupt ER homeostasis by promoting unfolded protein overload [9]. Unfolded protein response (UPR) is activated as an adaptive response to restore ER activity and maintain protein quality to ensure proper protein synthesis, secretion, and correct folding of protein [10-12]. When ER stress is severe and/or prolonged, it can shift toward apoptosis (cell death), although the mechanism for this transition is not well understood [10,13-15]. ER stress has been linked to multiple disorders ranging from neurodegenerative diseases to metabolic disorders such as obesity, insulin resistance, type 2 diabetes, and chronic inflammation $[9,10,16]$. Metabolically active tissue including adipose tissue is not excluded from the ER stress outcomes [17-19]. However, the consequences of ER stress on adipocyte functions including thermogenic capacity are poorly understood.

In adipose tissue, obesity results in chronic stress and dysfunction caused by the increased demand of synthetic machinery [17]. Several studies have shown a significant activation of ER stress in high-fat-induced obesity [20-22]. In addition, both brown and beige adipocyte activation stimulated by cold exposure was impaired in obese adipose tissue [23]. Adversely, a decrease in adaptive thermogenesis has also been suggested as a contributing factor to obesity [6]. As obesity is characterized by diverse metabolic symptoms such as ER stress and inflammation, there is still no direct link about how ER stress itself could regulate Ucp1 expression in beige adipocytes.

Previous study has shown that the activation of downstream signaling in ER stress, such as that involving inositol-requiring enzyme $1 \alpha$ (IRE1 $\alpha)$ and X-box binding protein 1 (XBP1), was needed for Ucp1 expression in BAT [16]. Further, the inactivation of ER stress in BAT, WAT, or macrophages resulted in an improved adaptive thermogenesis response [6,24], while treatment of chemical chaperones (ER stress inhibitor) could increase energy expenditure and activate browning of WAT [25-27]. These reports suggest a possible unknown regulatory mechanism of ER stress in the browning of WAT. In this study, we investigated the effect of ER stress stimulation on Ucp1, an adipocyte browning marker, in beige adipocytes.

\section{Results}

\subsection{ER Stress Decreases Ucp1 mRNA Level in Beige Adipocytes}

To investigate the regulation of ER stress on the thermogenic capacity of beige adipocytes, inguinal white adipose tissue (IWAT) cells were differentiated to beige adipocytes and treated with tunicamycin, an ER stress inducer. ER stress stimulation was confirmed by the upregulation of ER stress markers: binding immunoglobulin protein (Bip) and CCAAT-enhancer-binding protein homologous protein (Chop) (Figure 1A). Consequently, Ucp1 expression was severely suppressed (Figure 1A). The addition of chemical chaperone 4-phenylbutyrate (PBA) alleviated ER stress markers Bip and Chop, while also rescued $U c p 1$ expression (Figure $1 \mathrm{~A}$ ). These results indicate that ER stress suppressed $U c p 1$ expression in beige adipocytes.

Accumulating evidence has identified the cross-talk between UPR and the mitogen-activated protein kinase (MAPK) signaling pathway as a result of ER stress stimulation [13]. Indeed, we found extracellular signal-regulated kinase (ERK) and c-Jun N-terminal kinase (JNK), which are parts of the MAPK signaling pathway, were activated by tunicamycin treatment in our experimental condition. Both ERK (Figure 1B) and JNK (Figure 1C) were phosphorylated after ER stress stimulation. The addition of either ERK or JNK inhibitor (U0126 or SP600125, respectively) could ameliorate tunicamycin-induced suppression of Ucp1 expression (Figure 1D,E and Figure S1A,B) while inhibiting the phosphorylation of ERK (Figure 1F and Figure S1C) or JNK (Figure 1G and Figure S1D), indicating an ER stress-induced suppression of Ucp1 expression via the activation of ERK and JNK pathway. 


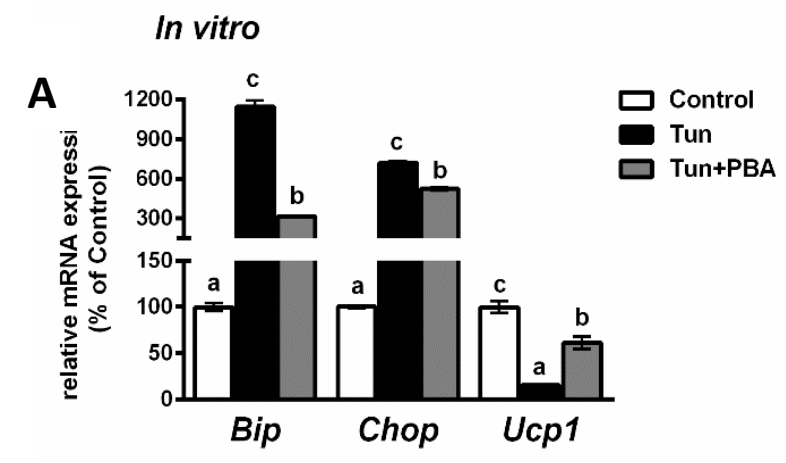

B

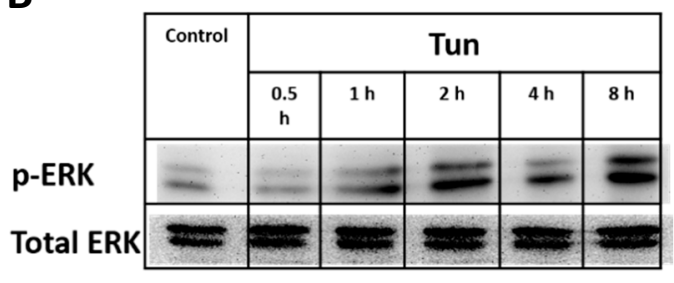

D

Ucp1

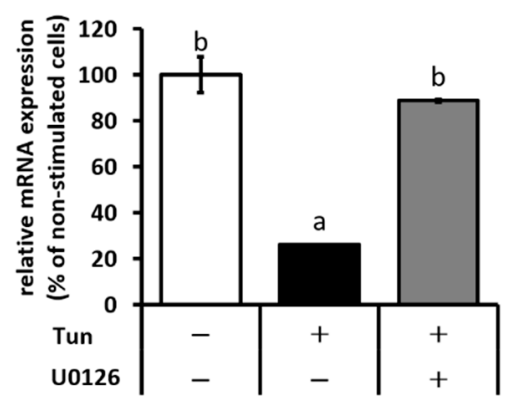

F

p-ERK

Total ERK
C

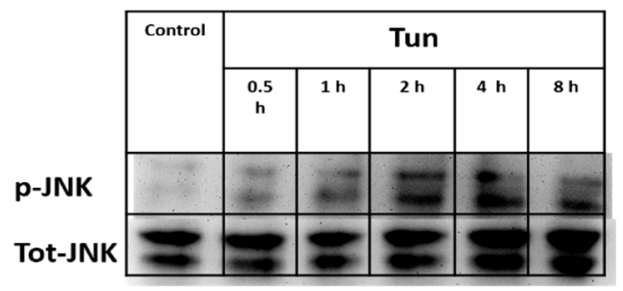

E

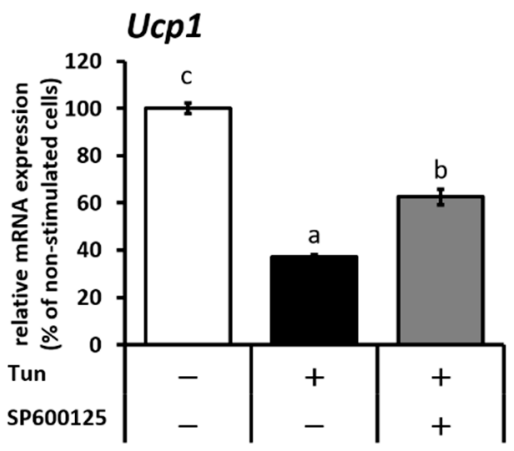

G

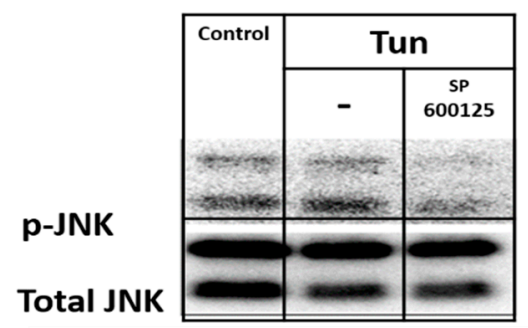

Figure 1. Endoplasmic reticulum (ER) stress stimulation downregulates uncoupling protein 1 (Ucp1) through phosphorylation of extracellular signal-regulated kinase (ERK) and c-Jun N-terminal kinase (JNK) in inguinal white adipose tissue (IWAT) cells. (A) the mRNA expression levels of ER stress markers: binding immunoglobulin protein (Bip), CCAAT-enhancer-binding protein homologous protein (Chop), and adipocyte browning markers Ucp 1 after treatment with $1 \mu \mathrm{M}$ tunicamycin (Tun) for $12 \mathrm{~h}$ with or without $20 \mathrm{mM}$ 4-phenylbutyrate (PBA) for $24 \mathrm{~h}$ before collection. Phosphorylation of (B) ERK and (C) JNK at different time points when treated with $1 \mu \mathrm{M}$ tunicamycin. (D,E) Ucp1 mRNA expression and phosphorylation of ERK (F) or JNK (G) after being treated with (D,F) 80 or $100 \mu \mathrm{M}$ ERK inhibitor (U0126) for $14 \mathrm{~h}$, or (E,G) $25 \mu \mathrm{M}$ JNK inhibitor (SP600125) for $13 \mathrm{~h}$, followed by tunicamycin $(1 \mu \mathrm{M} ; 12 \mathrm{~h}$ ). Data are presented as mean \pm S.E.M. (error bars). $n=4$ in each group. Different letters indicate significant differences $(p<0.05)$ according to one-way ANOVA followed by Tukey-Kramer multiple comparison test. 


\subsection{ER Stress Induces Downregulation of the Ucp1 Activator, Ppar $\gamma$, Preferably via the JNK Pathway}

It has been previously shown that Ppary is a direct target of both ERK and JNK, which may result in decreased Ppary transcriptional activity [28] and thus possibly affect Ucp1 expression. Alongside Ucp1 downregulation, the level of Ppar $\gamma$ mRNA was decreased upon tunicamycin treatment (Figure 2A,B) in IWAT cells but rescued when cells were treated with either an ERK (Figure 2A) or JNK inhibitor (Figure 2B). Besides that, ER stress markers (Bip, Chop and X-box binding protein 1 splicing (Xbp1s)) were partially recovered through ERK and JNK inhibition (Figure 2C,D), indicating the involvement of ERK/JNK pathway in regulating Pparr expression.

A

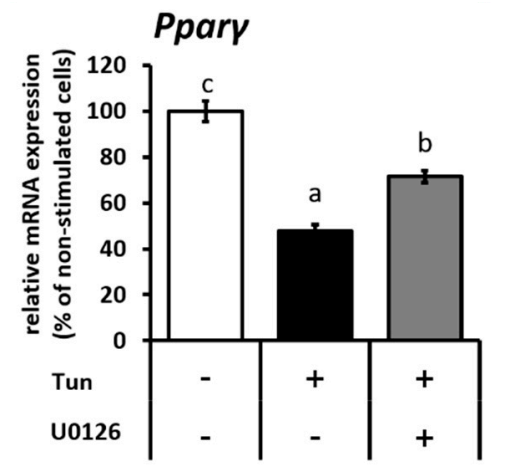

\section{C}

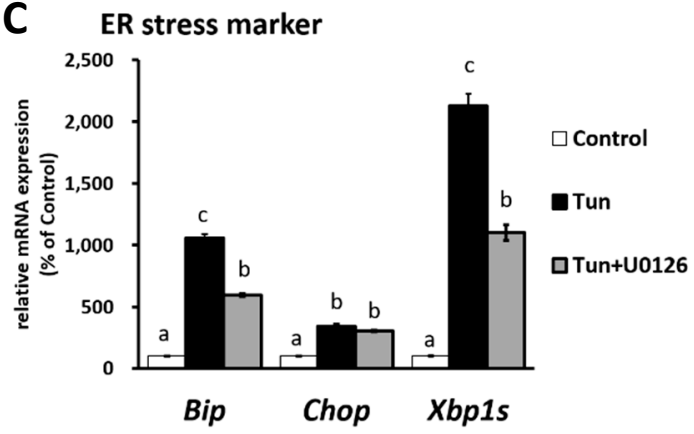

B

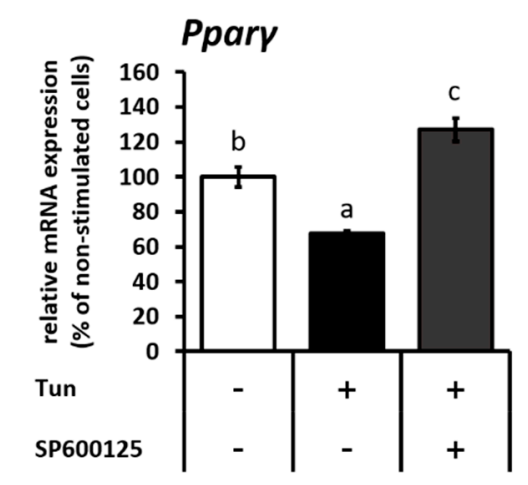

D

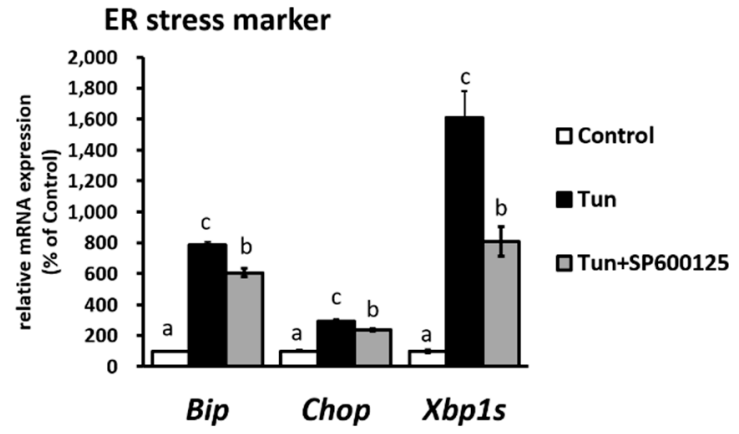

Figure 2. ER stress decreases Ucp 1 activator, peroxisome proliferator-activated receptor $\gamma($ Ppar $\gamma)$ mRNA expression through ERK and JNK pathway in IWAT cells. Ppar $\gamma$ and ER stress marker (Bip, Chop, and X-box binding protein 1 splicing-Xbp1s) mRNA expression in cells treated with $(\mathbf{A}, \mathbf{C})$ $100 \mu \mathrm{M}$ ERK inhibitor (U0126) for $14 \mathrm{~h}$ or (B,D) $25 \mu \mathrm{M}$ JNK inhibitor (SP600125) for $13 \mathrm{~h}$, followed by tunicamycin $(1 \mu \mathrm{M}, 12 \mathrm{~h})$. Data are presented as mean \pm S.E.M. (error bars). $n=4$ in each group. Different letters indicate significant differences $(p<0.05)$ according to one-way ANOVA followed by Tukey-Kramer multiple comparison test.

We further investigated the possibility of post-translational modifications of Ppar $\gamma$ through the phosphorylation in serine 112 (S112), which is one of the main targets of ERK/JNK [29]. However, we did not find any change in the level of phosphorylation; instead, the Ppar $\gamma$ protein level was significantly reduced (Figure 3A). A time-course experiment revealed that the decrease in the Ppary protein level was initiated earlier than the decrease in phosphorylation of Ppar $\gamma$ at S112 (Figure 3B). At this time $(\sim 4 \mathrm{~h})$, ERK and JNK were both activated (Figure 1B,C). However, only treatment with the JNK inhibitor (SP600125), but not the ERK inhibitor (U0126), could rescue Ppary protein levels (Figure $3 \mathrm{C}$ ), suggesting that JNK is the main pathway for the tunicamycin-induced reduction in Ppary protein. 
A

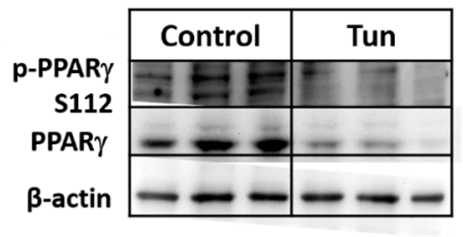

p-PPAR $\gamma$ S112/ PPAR $\gamma$

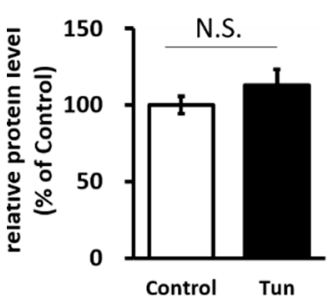

$\mathrm{PPAR} \gamma / \beta$-actin

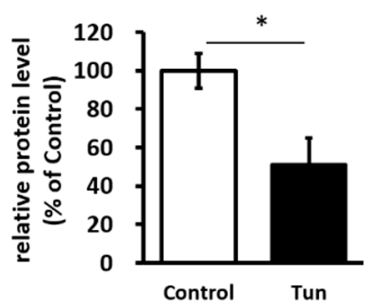

B

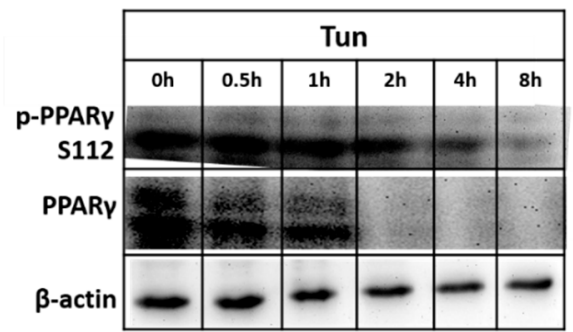

C

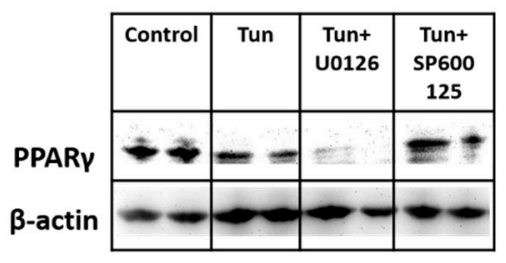

PPAR $\gamma / \beta$-actin

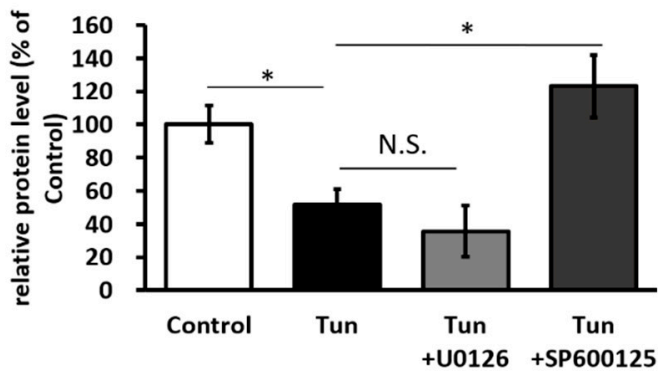

Figure 3. ER stress decreases Ppar $\gamma$ protein level preferably through JNK pathway in IWAT cells. Ppar $\gamma$ phosphorylation at serine 112 (S112) and Ppar $\gamma$ protein level in cells treated with $1 \mu \mathrm{M}$ tunicamycin (A) for $12 \mathrm{~h}$ or (B) at different time points. (C) Ppar $\gamma$ protein after treated with $100 \mu \mathrm{M}$ U0126 for $14 \mathrm{~h}$ or $25 \mu \mathrm{M}$ SP600125 for $13 \mathrm{~h}$, followed by tunicamycin $(1 \mu \mathrm{M}, 12 \mathrm{~h})$. $\beta$-actin was used as a loading control. Data are presented as mean \pm S.E.M. (error bars). $n=3-4$ in each group. ${ }^{*}$ indicates significant differences $(p<0.05)$ according to unpaired- $t$ test. N.S., not significant.

Next, to confirm the role of Ppary in ER stress-regulated Ucp1 expression, we treated IWAT cells with Ppary antagonist (GW9662). GW9662 similarly decreased Ucp1 expression as tunicamycin (Figure 4 and Figure S2). However, the addition of GW9662 in tunicamycin treatment did not enhance the suppression of $U_{c p} 1$ expression (Figure 4 and Figure S2), suggesting that ER stress-suppressed Ucp1 expression is indeed mediated through Ppar $\gamma$.

\subsection{ER Stress Stimulates Ppary Degradation that Leads to the Reduced Binding Activity}

Next, to investigate whether the decreased Ppar $\gamma$ protein was dependent on the decrease in its mRNA expression, we performed a cycloheximide chase experiment to measure Ppar $\gamma$ protein stability. Tunicamycin treatment seemed to accelerate Ppar $\gamma$ degradation under cycloheximide addition (Figure 5A). The half-life of Ppar $\gamma$ was significantly reduced from 5.9 to $3.5 \mathrm{~h}$ upon tunicamycin treatment (Figure 5B). However, the addition of proteasome inhibitor (lactacystin) could rescue Ppar $\gamma$ protein (Figure 5C), suggesting that the degradation of Ppar $\gamma$ was mediated via proteasome degradation. 


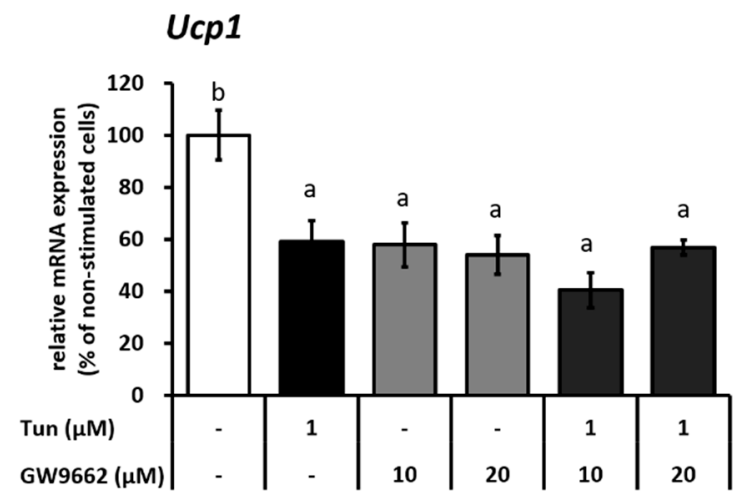

Figure 4. ER stress suppresses Ucp1 expression through the reduced activity of Ppar $\gamma$ in IWAT cells. Ppar $\gamma$ mRNA expression in cells treated with Ucp 1 mRNA expression after treatment with 10 or $20 \mu \mathrm{M}$ Ppary antagonist (GW9662) for $13 \mathrm{~h}$, followed by $1 \mu \mathrm{M}$ tunicamycin $(12 \mathrm{~h})$. Data are presented as mean \pm S.E.M. (error bars). $n=4$ in each group. Different letters indicate significant differences $(p<0.05)$ according to one-way ANOVA followed by Tukey-Kramer multiple comparison test.

To further examine the consequence of Ppary degradation for its role as activator, luciferase assay was done to analyze PPAR response element (PPRE) transcriptional activity under tunicamycin stimulation. Although it was in undifferentiated IWAT cells, a luciferase assay clearly showed that the tunicamycin treatment canceled the upregulation of PPRE transcriptional activity by rosiglitazone (Figure 5D). However, the stabilization of Ppary by lactacystin altered PPRE activity (Figure 5D), indicating that Ppar $\gamma$ degradation indeed affects its binding activity. To finally connect the decrease in Ppary binding activity to Ucp 1 expression, we performed chromatin immunoprecipitation (ChIP) assay to measure the recruitment of Ppar $\gamma$ onto the PPRE within the Ucp 1 enhancer region. As shown in Figure 5E, the recruitment level of Ppar $\gamma$ to Ucp1 promoter was significantly decreased after tunicamycin treatment. These data established that ER stress-induced Ppar $\gamma$ degradation was associated with the downregulated Ucp1 expression due to the loss of Ppar $\gamma$ activator binding to Ucp1 promoter.

\subsection{ER Stress Suppressed Both Ucp1 mRNA and Protein Expression in Adipose Tissue}

After observing a negative regulation of ER stress on Ucp 1 expression in vitro, we next investigated if the same phenomena also occur in vivo. Browning of WAT was induced by injecting Ppar $\gamma$ agonist (rosiglitazone) for 10 days. As shown by the expression level of ER stress marker genes Bip and Chop (Figure 6A), the rosiglitazone treatment had no effect on ER stress. IWAT, known as the most typical browning-stimulated WAT, was confirmed to undergo browning through a significant increase in Ucp $1 \mathrm{mRNA}$ level (Figure 6A) after rosiglitazone treatment. After beige adipocytes were developed in IWAT, ER stress was afflicted through tunicamycin treatment. A single injection of tunicamycin could stimulate ER stress in IWAT as indicated by elevated Bip and Chop (Figure 6A) mRNA levels. In this state, tunicamycin canceled $U c p 1$ mRNA upregulation by rosiglitazone to basal levels (Figure 6A). However, similar to in vitro result, ERK and JNK inhibition could ameliorate the tunicamycin-suppressed $U_{c p} 1$ expression ex vivo (Figure 6B). It is important to note that ERK and JNK inhibition barely altered ER stress marker (Bip, Chop, and Xbp1s) (Figure 6B), suggesting that the alteration of ERK and JNK pathway possibly only affect the downstream signaling of UPR, which in this case was Ucp1 regulation. 


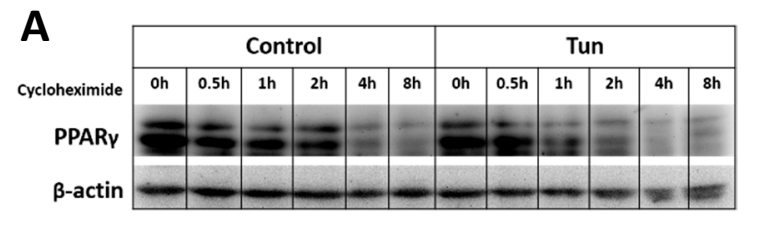

C
B

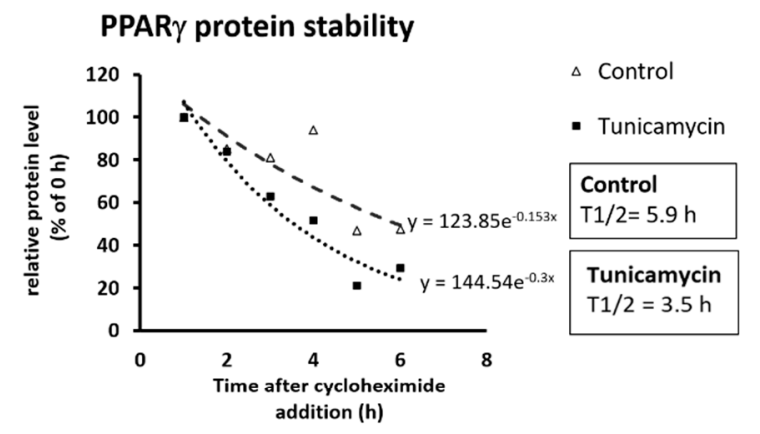

D

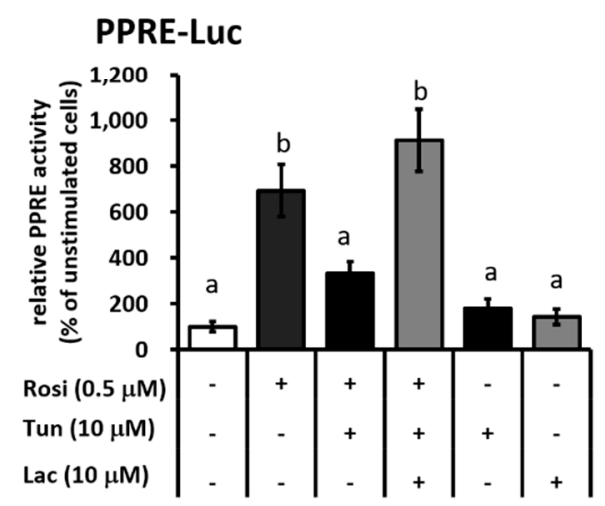

E

ChIP: Ucp1 Enhancer

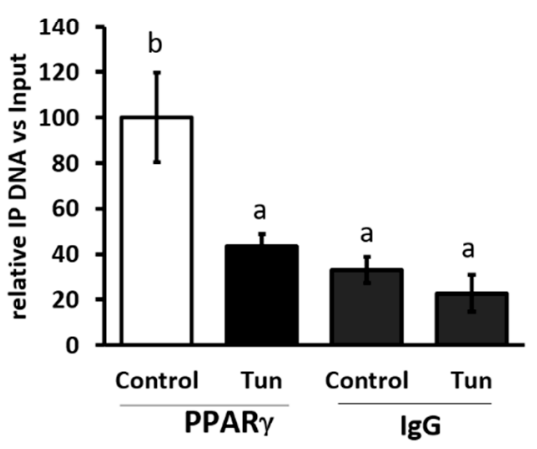

Figure 5. ER stress induces Ppar $\gamma$ degradation and reduces its binding activity in IWAT cells. (A) Ppar $\gamma$ protein level in cells during the cycloheximide chase experiment $(20 \mu \mathrm{g} / \mathrm{mL})$ at different time points with or without $1 \mu \mathrm{M}$ tunicamycin treatment. (B) Regression analysis of Ppar $\gamma$ protein stability of (A). (C) Ppar $\gamma$ protein level after treated with $1 \mu \mathrm{M}$ tunicamycin and/or $10 \mu \mathrm{M}$ proteasome inhibitor, lactacystin (Lac) for $4 \mathrm{~h}$ in the presence of cycloheximide $(20 \mu \mathrm{g} / \mathrm{mL})$. $\beta$-actin was used as a loading control. (D) PPAR response element (PPRE) transcriptional activity in undifferentiated IWAT cells after treated with $0.5 \mu \mathrm{M}$ rosiglitazone (Rosi) for $24 \mathrm{~h}, 10 \mu \mathrm{M}$ tunicamycin and/or $10 \mu \mathrm{M}$ lactacystin for $4 \mathrm{~h}$, based on a luciferase assay. (E) Ppar $\gamma$ recruitment level in the Ucp1 distal enhancer region analyzed by chromatin immunoprecipitation (ChIP) assay after cells were treated with $1 \mu \mathrm{M}$ tunicamycin. IgG was used as a mock control. Data are presented as mean \pm S.E.M. (error bars). $n=3-5$ in each group. Different letters indicate significant differences $(p<0.05)$ according to one-way ANOVA followed by Tukey-Kramer multiple comparison test.

As mRNA expression was reduced, the Ucp1 protein level was subsequently decreased under ER stress stimulation (Figure 6C and Figure S3A). The Ucp1 downregulation was also observed via immunohistochemistry (IHC) of Ucp1 (Figure 6D) in IWAT. Hematoxylin and eosin (H\&E) staining showed an increasing size of lipid droplet after tunicamycin injection (Figure 6D and Figure S4), indicating a reversal of browning (whitening). These data demonstrated that ER stress is a strong negative regulator of Ucp1 expression in IWAT. Following the in vitro result, we also found a significant reduction in Ppar $\gamma$ protein levels in vivo (Figure 7 and Figure S3B), but not mRNA levels (Figure 6A), suggesting the decreased Ppar $\gamma$ protein as the main factor for Ucp1 downregulation during ER stress stimulation. 
A

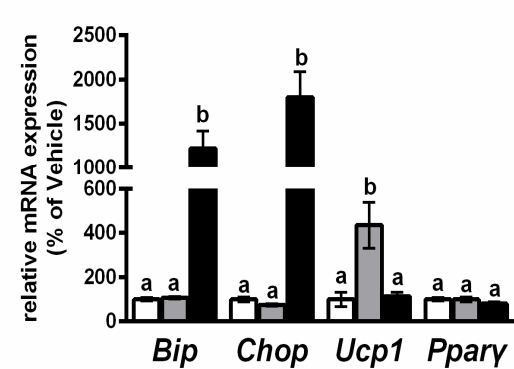

C

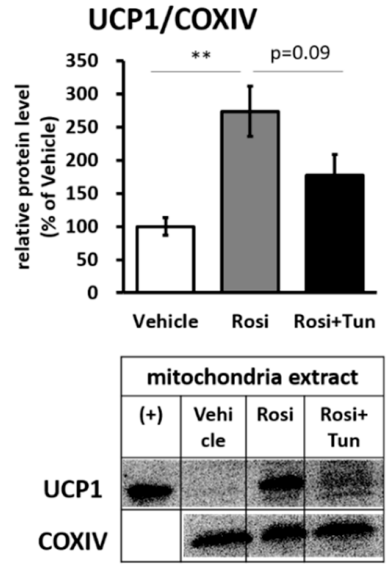

B

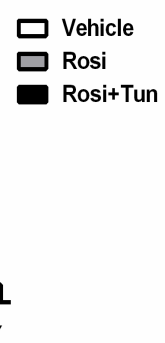

\section{Ex vivo}

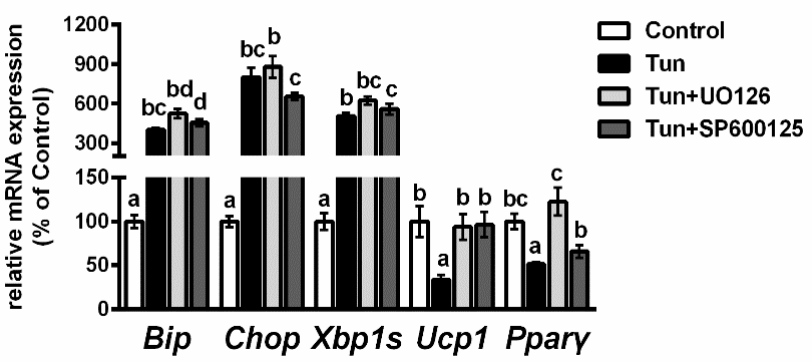

D

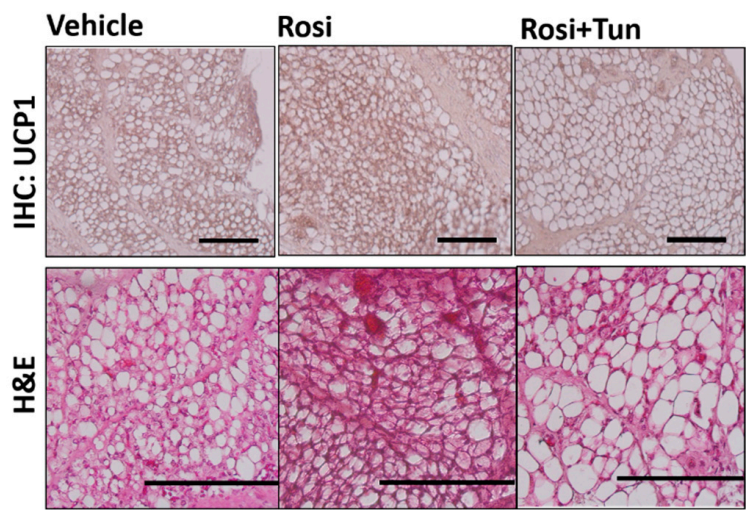

Figure 6. ER stress stimulation negatively regulates $U c p 1$ expression in IWAT in vivo and ex vivo. (A) The mRNA expression levels of ER stress markers Bip, Chop, and Xbp1s, and adipocytes browning markers Ucp 1 and Ppary in (A) IWAT in vivo of mice injected with vehicle, $10 \mathrm{mg} / \mathrm{kg}$ rosiglitazone for 10 days (Rosi), or rosiglitazone and $10 \mathrm{mg} / \mathrm{kg}$ tunicamycin (Rosi+Tun) for the last $24 \mathrm{~h}$ or (B) IWAT ex vivo after treated with $1 \mu \mathrm{M}$ tunicamycin for $24 \mathrm{~h}$ with or without $100 \mu \mathrm{M} \mathrm{U} 0126$ for $26 \mathrm{~h}$ and $25 \mu \mathrm{M}$ SP600125 for $25 \mathrm{~h}$. (C) Ucp1 protein level, and (D) immunohistochemical staining (IHC) of Ucp1 (scale bar $200 \mu \mathrm{M}$ ) as well as hematoxylin and eosin (H\&E) staining (scale bar $500 \mu \mathrm{M}$ ) in IWAT in vivo. $(+)$ indicates the positive control (brown adipose tissue). Cytochrome c oxidase subunit IV (COXIV) were used as loading controls. Data are presented as mean \pm S.E.M. (error bars). $n=3-8$ in each group. Different letters indicate significant differences $(p<0.05)$ according to one-way ANOVA followed by Tukey-Kramer multiple comparison test. ${ }^{* *}$ indicates significant differences $(p<0.01)$ according to unpaired- $t$ test. N.S., not significant.

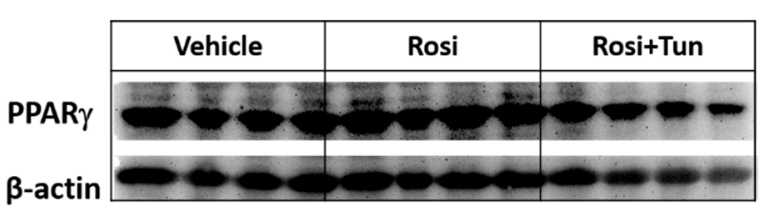

$\beta$-actin

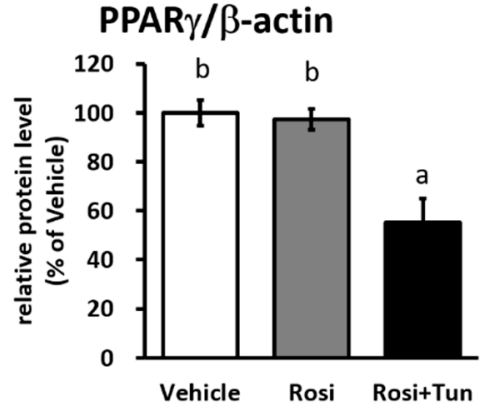

Figure 7. ER stress reduced Ppar $\gamma$ protein in IWAT. Ppar $\gamma$ protein level in IWAT of mice injected with vehicle, $10 \mathrm{mg} / \mathrm{kg}$ rosiglitazone for 10 days (Rosi), or rosiglitazone and $10 \mathrm{mg} / \mathrm{kg}$ tunicamycin (Rosi+Tun) for the last $24 \mathrm{~h}$. $\beta$-actin were used as loading controls. Data are presented as mean \pm S.E.M. (error bars). $n=3-8$ in each group. Different letters indicate significant differences $(p<0.05)$ according to one-way ANOVA followed by Tukey-Kramer multiple comparison test. 


\section{Discussion}

ER stress and inflammation have been known as a hallmark of metabolic syndrome. While how inflammation could defect Ucp1 expression in adipose tissue has been reported [23,30], the effect of ER stress in Ucp1 regulation is still unclear. However, as shown in Figure 6C, Ucp1 protein level was not detected in the basal level of IWAT (without rosiglitazone). Subsequently, Ucp1 protein level remained undetected when ER stress was induced by tunicamycin (Figure S3A). Therefore, we decided to investigate the effect of ER stress specifically in beige adipocytes. We first needed to induce the formation of beige adipocytes in IWAT by using drug-induced BAT activation. Among those, $\beta 3$-adrenergic receptor agonist (such as isoproterenol) and the activators of Ppary (such as rosiglitazone) have been widely used for treating obesity and type 2 diabetes through the browning-related modification in carbohydrate and lipid metabolism [31,32]. Isoproterenol and rosiglitazone themselves have been reported to increase the levels of $U c p 1$ mRNA in adipocytes, thus both drugs can be used to induce beige adipocytes [31,33-35]. However, isoproterenol is reported to stimulate ER stress [36] while activating $U_{c p} 1$, as confirmed in our preliminary experiment (data not shown), therefore using isoproterenol did not fit this study. Unlike isoproterenol, rosiglitazone could induce browning in beige adipocytes without afflicting ER stress, which is shown in this report. Hence, the use of rosiglitazone would not hinder the main ER stress stimulator from tunicamycin. It is also important to note that the long treatment of rosiglitazone has been reported to have adverse effects such as weight gain (due to fluid retention) and the expansion of adipose tissue in many studies [37]. However, we have confirmed that our experimental model was done with no side effect in body weight (data not shown) or adipose tissue expansion (Figure 6D).

Ppary has been widely known as an important activator that could influence Ucp1 expression $[1,38,39]$. Interestingly, we found that Ppar $\gamma$ protein level is decreased under ER stress stimulation even at the basal level ( s3 B). Thus, there is a possibility that ER stress-reduced Ppar $\gamma$ protein would further affect Ucp1 expression and prevent WAT to undergo browning. Although this possibility could not be proven due to low Ucp1 detection in WAT, our study clearly shows that ER stress was indeed a strong negative regulator of both Ucp 1 mRNA and protein expression in beige adipocytes. Furthermore, ER stress stimulation seemed to increase the lipid droplet size even in the activated beige adipocytes, which indicates whitening (reversal of browning) as shown in this study, suggesting ER stress may closely involve to the impaired browning.

To find the mechanism under ER stress-suppressed Ucp1 expression, we first noticed the major involvement of MAPK pathways: ERK and JNK as a part of UPR during ER stress. Among the three ER stress sensors that have been established, IRE1 activation has been reported to promote the phosphorylation of ERK and JNK [13,20,40], which was also shown in this study. However, the consequences of ERK and JNK activation to Ucp1 expression during ER stress is unknown. Herein, we found that both ERK and JNK inhibition could recover Ucp1_expression under ER stress condition, suggesting that ER stress suppressed-Ucp 1 expression might be mediated through ERK and JNK pathways. Furthermore, Ppary regulation by ERK and JNK during ER stress stimulation seemed to have similar pattern as Ucp1. Following that, Ppar $\gamma$ protein level was also decreased by tunicamycin treatment. However, only JNK inhibition could rescue Ppary protein level. These results suggesting that the reduced Ucp 1 expression was mediated preferably through JNK-Ppary interaction.

It has been known that Ppary is a phosphorylation target of MAPK which includes ERK and

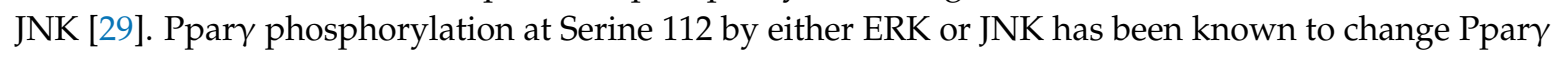
conformation that lead to the protein degradation and repressed transcriptional activation $[29,41]$. However, to our surprise, we did not find significant change in phosphorylation of Ppar $\gamma$. Instead, Ppary protein level was decreased earlier than its phosphorylation. Thus, we assume that although JNK-mediated the reduction of Ppar $\gamma$ activity by phosphorylation has been established through several studies [29,41,42], the detail mechanism may be different depending on the source of JNK activation. In the case of ER stress, JNK might mediate the decrease of Ppar $\gamma$ mRNA and protein level independent of phosphorylation modification. Accordingly, JNK activation is also known to be associated with 
apoptosis through promoting phosphorylation and activation of pro-apoptotic protein Bcl-2-associated $\mathrm{X}$ protein (Bax) $[13,43,44]$. Indeed, we found that chronic ER stress (12-20 h) in our experimental condition induced cell apoptosis, indicated by the upregulated Chop expression, decreased cell viability (Figure S5A), and increased in cleavage Caspase 3 (Figure S5B,C). Chop has been reported to participate in cell death program during ER stress [45]. On the other hand, Caspase 3 (apoptosis marker) has been found to mediate Ppar $\gamma$ cleavage [46]. These pieces of evidence suggest that JNK-mediated reduced Ppary activity may be related to the cell apoptosis response.

Regardless, we found a consistent decrease in Ppar $\gamma$ activity in vitro, ex vivo, and in vivo after ER stress stimulation. Indeed, when we reduced Ppar $\gamma$ activity by Ppar $\gamma$ antagonist (GW9662), it similarly downregulated Ucp 1 expression as tunicamycin. Furthermore, the co-treatment between tunicamycin and GW9662 did not enhance the suppression of $U c p 1$ expression, suggesting that both compounds regulated Ucp1 expression preferably through the same pathway, which in this case was through Ppar $\gamma$. To finally connect the consequence of Ppar $\gamma$ reduction to Ucp 1 expression, we investigated the PPRE activity under tunicamycin treatment. Ucp 1 is one of the established activation target of Ppar $\gamma$ in adipose tissue, equipped with PPRE binding sequence located on the Ucp 1 distal enhancer (-2494 to -2318 bp) [1]. Herein, we showed that PPRE activity was significantly affected after ER stress stimulation, based on the luciferase assay. Although it was performed in undifferentiated IWAT cells due to the low transfection efficiency in differentiated cells, we further confirmed in ChIP assay that Ppar $\gamma$ recruitment to Ucp1 promoter was consistently reduced in differentiated IWAT cells. These pieces of evidence thus show that the reduced Ppar $\gamma$ binding to Ucp 1 promoter are likely due to the decreased protein level and subsequently affected Ucp 1 mRNA downregulation as ER stress was stimulated.

While the reduced Ppar $\gamma$ mRNA expression was only seen in vitro, the Ppar $\gamma$ protein level was decreased both in vitro and in vivo. These results might indicate that the loss of Ppar $\gamma$ protein was not dependent on its mRNA expression. Later, we found that Ppar $\gamma$ protein stability was disturbed by ER stress stimulation, suggesting that Ppar $\gamma$ protein was degraded. In addition, it has been known that ER stress induction could lead to the activation of protein degradation pathway to remove the accumulation of unfolded protein [14]. Basically, there are two major protein degradation pathways: proteasomes (via ER-associated degradation or ERAD) and lysosome (via autophagy) degradation [14,47]. Between two pathways, ERAD is recognized as the predominant cellular mechanism for removal of unfolded protein [14,48]. Certainly, the addition of proteasome inhibitor (lactacystin) rescued Ppary protein and PPRE activity, suggesting proteasome-mediated Ppar $\gamma$ degradation affects its binding activity to $U c p 1$ promoter during ER stress stimulation in beige adipocytes.

Interestingly, the induction of ER stress did not only affect Ucp 1 mRNA expression, but also Ucp1 protein level. Although, it might be linear to the decreased in mRNA expression, there still has possibility that the reduced Ucp1 protein level was affected directly through ERAD mechanism. Therefore, further investigation is needed to carefully assess Ucp1 protein modification in ER stress. Regardless, this study presents a novel evidence about the negative regulation of ER stress signaling to Ucp1 expression via the suppression of activator Ppar $\gamma$ in beige adipocytes.

\section{Materials and Methods}

\subsection{Materials}

All chemicals were obtained from Nacalai Tesque (Kyoto, Japan), Wako (Osaka, Japan), Corning (Corning, NY, USA), Qiagen (Hilden, Germany), Invitrogen (Carlsbad, CA, USA), and Sigma-Aldrich (St. Louis, MO, USA). Tunicamycin, U0126, SP600125, and cycloheximide were purchased from Nacalai Tesque (Kyoto, Japan). GW9662 (Sigma-Aldrich, St. Louis, MO, USA), rosiglitazone (LKT Laboratories, St. Paul, MN, USA), and PBA (Santa Cruz Biotechnology, Dallas, TX, USA) were acquired from the indicated companies. 


\subsection{Animal Experiment}

Mice were kept in a temperature-controlled room at $23 \pm 1{ }^{\circ} \mathrm{C}$ with a $12 \mathrm{~h}$ light/dark cycle and free access to food (standard diet) and water. To stimulate browning, 6-10-week-old male C57BL/6J mice (SLC, Shizuoka, Japan) were intraperitoneally injected with $10 \mathrm{mg} / \mathrm{kg}$ rosiglitazone daily for 10 days [49]. On Day 10,10 mg/kg tunicamycin was injected to induce ER stress. Mice were then fasted overnight. Twenty-four hours after the last injection, mice were sacrificed, and IWAT was harvested for mRNA and protein analysis. The mice were handled in accordance with procedures approved by the Kyoto University Animal Care Committee (Permission number: 29-62, 20 April 2012).

\subsection{Ex Vivo Experiment}

To stimulate browning, 6-week-old male C57BL/6J mice (SLC, Shizuoka, Japan) were intraperitoneally injected with $10 \mathrm{mg} / \mathrm{kg}$ rosiglitazone daily for 10 days [49]. Twenty-four hours after last injection, IWAT was collected and immediately incubated in serum free medium containing $1 \mu \mathrm{M}$ tunicamycin for $24 \mathrm{~h}$ with or without $100 \mu \mathrm{M}$ ERK inhibitor (U0126) for $26 \mathrm{~h} / 25 \mu \mathrm{M}$ JNK inhibitor (SP600125) for $25 \mathrm{~h}$. IWAT was then extracted for mRNA analysis.

\subsection{Cell Culture}

Immortalized primary IWAT cells were kindly provided by Dr. S. Kajimura (University of California, San Francisco, CA, USA). IWAT cells were maintained in a humidified $5 \% \mathrm{CO}_{2}$ atmosphere at $37^{\circ} \mathrm{C}$ using basic medium (DMEM) supplemented with $10 \%$ fetal bovine serum and 1\% penicillin/streptomycin. To induce the differentiation into beige adipocytes [49], two days post-confluent IWAT cells were stimulated with $0.5 \mathrm{mM}$ 1-methyl-3-isobutylxanthine, $2 \mu \mathrm{g} / \mathrm{mL}$ dexamethasone, $10 \mu \mathrm{g} / \mathrm{mL}$ insulin, $1 \mathrm{nM}$ triiodo-L-thyronine (T3), $0.5 \mu \mathrm{M}$ rosiglitazone (rosi), and $125 \mu \mathrm{M}$ indomethacin for $48 \mathrm{~h}$. The media was then replaced by basic medium containing $5 \mu \mathrm{g} / \mathrm{mL}$ insulin, $1 \mathrm{nM} \mathrm{T}$, and $0.5 \mu \mathrm{M}$ rosi every 2 days. Generally, the differentiation process took 8-10 days. ER stress was induced by the addition of tunicamycin $(1 \mu \mathrm{M})$ in serum-free medium for $12 \mathrm{~h}$, unless mentioned.

\subsection{RNA Preparation and Quantification of Gene Expression}

RNA was extracted as described previously [50]. Total RNA was collected from cultured cells or tissues using Sepasol-RNA I Super G (Nacalai Tesque, Kyoto, Japan) or QIAzol Lysis Reagent (Qiagen, Hilden, Germany), respectively. RNA expression was quantified by real-time PCR using a LightCycler System (Roche Diagnostics, Mannheim, Germany) with SYBR green fluorescence signal detection. All mRNA signals were normalized to a 3664 internal control. The primer sequences are listed in Table 1 .

Table 1. Primers used for RNA quantification.

\begin{tabular}{|c|c|c|}
\hline Gene & Forward & Reverse \\
\hline Ucp1 & 5'-CAAAGTCCGCCTTCAGATCC-3' & 5'-AGCCGGCTGAGATCTTGTTT-3' \\
\hline Ppary & 5'-GGAGATCTCCAGTGATATCGACCA-3' & 5'-ACGGCTTCTACGGATCGAAAACT-3' \\
\hline $36 b 4$ & 5'-TCCTTCTTCCAGGCTTTGGG-3' & 5'-GACACCCTCCAGAAAGCGAG-3' \\
\hline Bip & 5'-GTTTGCTGAGGAAGACAAAAAGCTC-3' & 5'-CACTTCCATAGAGTTTGCTGATAAT-3' \\
\hline Chop & 5'-GTCCAGCTGGGAGCTGGAAG-3' & 5'-CTGACTGGAATCTGGAGAG-3' \\
\hline
\end{tabular}

\subsection{Protein Extraction and Western Blotting}

Western blotting was performed as previously described [50]. The protein concentration was measured using the DC protein assay (Bio-Rad, Hercules, CA, USA). The primary antibodies included anti-Ucp1 (Sigma-Aldrich, St. Louis, MO, USA), anti-COXIV, anti-phospho-p44/42 MAPK (ERK1/2), anti-phospho-SAPK/JNK (Thr183/Tyr185), anti-SAPK/JNK, anti-Ppar $\gamma$, and anti- $\beta$-actin (all purchased from Cell Signaling Technology, Danvers, MA, USA). The secondary antibody staining was visualized using a chemiluminescent horseradish peroxidase substrate (Millipore, Burlington, MA USA). 


\subsection{Chromatin Immunoprecipitation (ChIP) Assay}

The ChIP assay was performed as described previously [50]. The cells were subjected to overnight immunoprecipitation with $8 \mu \mathrm{g}$ Ppary antibody (Perseus Proteomics, Tokyo, Japan), or rabbit IgG isotype control (Novus Biological, Littleton, CO, USA) as a mock control and analyzed by real-time PCR. The primer sequences are listed in Table 2.

Table 2. Primers used in ChIP assay.

\begin{tabular}{ccc}
\hline Gene & Forward & Reverse \\
\hline Ucp1 enhancer & 5'-CTCCTCTACAGCGTCACAGAGG-3' & 5-AGTCTGAGGAAAGGGTTGA-3' \\
\hline
\end{tabular}

\subsection{Luciferase Assay}

The luciferase assay was performed according to company protocol (Invitrogen, Carlsbad, CA, USA). Undifferentiated IWAT cells were transfected with plasmid containing the reporter vector driven by PPAR response element (PPRE-Luc) and a Ppar $\gamma$ expression vector using Lipofectamine 2000 reagent (Invitrogen, Carlsbad, CA, USA). The transfected cells were treated with $0.5 \mu \mathrm{M}$ rosiglitazone for $24 \mathrm{~h}$ and $10 \mu \mathrm{M}$ tunicamycin with or without $10 \mu \mathrm{M}$ lactacystin for $4 \mathrm{~h}$.

\subsection{Statistical Analysis}

All data were analyzed using Student's $t$-test or one-way ANOVA followed by Tukey-Kramer test, when variances were heterogeneous. All data are presented as means \pm SEM. Differences were considered significant at $p<0.05$.

\section{Conclusions}

The present study highlights a novel mechanism of ER stress regulation to Ucp1 expression preferentially via the suppression of activator Ppar $\gamma$ in beige adipocytes. Furthermore, it may provide a solid foundation about the involvement of ER stress to the impaired thermogenic capacity, especially in obese adipose tissue. Thus, recovering ER stress is proven essential to rescue thermogenic activity in adipose tissue.

Supplementary Materials: Supplementary Materials can be found at http:/ /www.mdpi.com/1422-0067/20/2/274/s1. Figure S1: The effect of extracellular signal-regulated kinase (ERK) and c-Jun N-terminal kinase (JNK) inhibitor in beige adipocytes; Figure S2: The effect of peroxisome proliferator-activated receptor gamma (Ppar $\gamma$ ) antagonist treatment in beige adipocytes; Figure S3: The effect of ER stress induction in IWAT; Figure S4: The lipid size of IWAT after ER stress stimulation; Figure S5: ER stress stimulation induces cell death program in beige adipocytes.

Author Contributions: Conceptualization, A.Y., A.D., and T.G.; Methodology, A.Y., A.D., H.-F.J., and J.K.; Analysis, A.Y. and A.D.; Writing-Original Draft Preparation, A.Y.; Writing-Review and Editing, H.-F.J., W.N., H.T., T.A., and T.G.; Supervision, H.-F.J., W.N., H.T., T.A., T.K., and T.G.; and Funding Acquisition, T.K. and T.G.

Acknowledgments: This study was supported by Grants-in-Aid for Scientific Research from the Ministry of Education, Culture, Sports, Science and Technology of Japan (16K07734 and 16H02551). The authors thank M. Komori and R. Yoshii for kindly providing technical assistance and secretarial support. We also appreciate S. Kajimura for providing the IWAT cells line. A.Y. was supported by Indonesia Endowment Fund for Education (LPDP) by Ministry of Finance, Indonesia. J.K. was supported by Monbukagakusho/MEXT, Japan.

Conflicts of Interest: The authors declare no conflict of interest.

\section{Abbreviations}

$\begin{array}{ll}\beta \text {-AR } & \text { beta-adrenergic receptor } \\ \text { BAT } & \text { brown adipose tissue } \\ \text { Bip } & \text { binding immunoglobulin protein } \\ \text { ChIP } & \text { chromatin immunoprecipitation } \\ \text { Chop } & \text { CCAAT-enhancer-binding protein homologous protein } \\ \text { COXIV } & \text { cytochrome c oxidase subunit IV }\end{array}$




$\begin{array}{ll}\text { ER } & \text { endoplasmic reticulum } \\ \text { ERAD } & \text { ER-associated degradation } \\ \text { ERK } & \text { extracellular signal-regulated kinase } \\ \text { H\&E } & \text { hematoxylin and eosin } \\ \text { IHC } & \text { immunohistochemical staining } \\ \text { IRE1 } \alpha & \text { inositol-requiring enzyme 1 alpha } \\ \text { IWAT } & \text { inguinal white adipose tissue } \\ \text { JNK } & \text { c-Jun N-terminal kinase } \\ \text { MAPK } & \text { mitogen-activated protein kinase } \\ \text { PBA } & \text { 4-phenylbutyrate } \\ \text { Ppar } \gamma & \text { peroxisome proliferator-activated receptor gamma } \\ \text { PPRE } & \text { PPAR response element } \\ \text { Rosi } & \text { rosiglitazone } \\ \text { S112 } & \text { serine 112 } \\ \text { T3 } & \text { triiodo-L-thyronine } \\ \text { Ucp1 } & \text { uncoupling protein } 1 \\ \text { WAT } & \text { white adipose tissue } \\ \text { XBP1s } & \text { X-box binding protein } 1 \text { splicing }\end{array}$

\section{References}

1. Villarroya, F.; Iglesias, R.; Giralt, M. PPARs in the control of uncoupling proteins gene expression. PPAR Res. 2007, 2007. [CrossRef] [PubMed]

2. Harms, M.; Seale, P. Brown and beige fat: Development, function and therapeutic potential. Nat. Med. 2013, 19, 1252-1263. [CrossRef] [PubMed]

3. Cannon, B.; Nedergaard, J. Brown adipose tissue: Function and physiological significance. Physiol. Rev. 2004, 84, 277-359. [CrossRef] [PubMed]

4. Vitali, A.; Murano, I.; Zingaretti, M.C.; Frontini, A.; Ricquier, D.; Cinti, S. The adipose organ of obesity-prone C57BL/6J mice is composed of mixed white and brown adipocytes. J. Lipid Res. 2012, 53, 619-629. [CrossRef] [PubMed]

5. Kalinovich, A.V.; de Jong, J.M.A.; Cannon, B.; Nedergaard, J. UCP1 in adipose tissues: Two steps to full browning. Biochimie 2017, 134, 127-137. [CrossRef] [PubMed]

6. Okla, M.; Wang, W.; Kang, I.; Pashaj, A.; Carr, T.; Chung, S. Activation of Toll-like receptor 4 (TLR4) attenuates adaptive thermogenesis via endoplasmic reticulum stress. J. Biol. Chem. 2015, 290, 26476-26490. [CrossRef]

7. Lee, Y.K.; Cowan, C.A. White to brite adipocyte transition and back again. Nat. Cell Biol. 2013, 15, 568-569. [CrossRef]

8. Rosenwald, M.; Perdikari, A.; Rülicke, T.; Wolfrum, C. Bi-directional interconversion of brite and white adipocytes. Nat. Cell Biol. 2013, 15, 659-667. [CrossRef]

9. Ozcan, U.; Cao, Q.; Yilmaz, E.; Lee, A.-H.; Iwakoshi, N.N.; Ozdelen, E.; Tuncman, G.; Gorgun, C.; Glimcher, L.H.; Hostamisligil, G.S. Endoplasmic Reticulum Stress Links Obesity, Insulin Action, and Type 2 Diabetes. Science 2003, 299, 1033-1036. [CrossRef]

10. Lindholm, D.; Korhonen, L.; Eriksson, O.; Kõks, S. Recent Insights into the Role of Unfolded Protein Response in ER Stress in Health and Disease. Front. Cell Dev. Biol. 2017, 5, 1-16. [CrossRef]

11. Walter, P.; Ron, D. The Unfolded Protein Response: From Stress Pathway to Homeostatic Regulation. Science 2011, 334, 1081-1085. [CrossRef]

12. Hetz, C.; Chevet, E.; Harding, H.P. Targeting the unfolded protein response in disease. Nat. Rev. Drug Discov. 2013, 12, 703-719. [CrossRef]

13. Darling, N.J.; Cook, S.J. The role of MAPK signalling pathways in the response to endoplasmic reticulum stress. Biochim. Biophys. Acta 2014, 1843, 2150-2163. [CrossRef]

14. Sano, R.; Reed, J.C. ER stress-induced cell death mechanisms. Biochim. Biophys. Acta 2013, 1833, 3460-3470. [CrossRef]

15. Tabas, I.; Ron, D. Integrating the mechanisms of apoptosis induced by endoplasmic reticulum stress. Nat. Cell Biol. 2011, 13, 184-190. [CrossRef] 
16. Kawasaki, N.; Asada, R.; Saito, A.; Kanemoto, S.; Imaizumi, K. Obesity-induced endoplasmic reticulum stress causes chronic inflammation in adipose tissue. Sci. Rep. 2012, 2, 1-7. [CrossRef]

17. Gregor, M.F.; Hotamisligil, G.S. Thematic review series: Adipocyte Biology. Adipocyte stress: The endoplasmic reticulum and metabolic disease. J. Lipid Res. 2007, 48, 1905-1914. [CrossRef]

18. Han, J.; Kaufman, R.J. The role of ER stress in lipid metabolism and lipotoxicity. J. Lipid Res. 2016, 57, 1329-1338. [CrossRef]

19. Bartelt, A.; Widenmaier, S.B.; Schlein, C.; Johann, K.; Goncalves, R.L.S.; Eguchi, K.; Fischer, A.W.; Parlakgül, G.; Snyder, N.A.; Nguyen, T.B.; et al. Brown adipose tissue thermogenic adaptation requires Nrf1-mediated proteasomal activity. Nat. Med. 2018, 24, 292-303. [CrossRef]

20. Salvadó, L.; Palomer, X.; Barroso, E.; Vázquez-Carrera, M. Targeting endoplasmic reticulum stress in insulin resistance. Trends Endocrinol. MeTable 2015, 26, 438-448. [CrossRef]

21. Alcalá, M.; Calderon-Dominguez, M.; Bustos, E.; Ramos, P.; Casals, N.; Serra, D.; Viana, M.; Herrero, L. Increased inflammation, oxidative stress and mitochondrial respiration in brown adipose tissue from obese mice. Sci. Rep. 2017, 7, 1-12. [CrossRef]

22. Deng, J.; Liu, S.; Zou, L.; Xu, C.; Geng, B.; Xu, G. Lipolysis response to endoplasmic reticulum stress in adipose cells. J. Biol. Chem. 2012, 287, 6240-6249. [CrossRef]

23. Sakamoto, T.; Nitta, T.; Maruno, K.; Yeh, Y.-S.; Kuwata, H.; Tomita, K.; Goto, T.; Takahashi, N.; Kawada, T. Macrophage infiltration into obese adipose tissues suppresses the induction of UCP1 expression in mice. Am. J. Physiol. 2016. [CrossRef]

24. Shan, B.; Wang, X.; Wu, Y.; Xu, C.; Xia, Z.; Dai, J.; Shao, M.; Zhao, F.; He, S.; Yang, L.; et al. The metabolic ER stress sensor IRE1 $\alpha$ suppresses alternative activation of macrophages and impairs energy expenditure in obesity. Nat. Immunol. 2017, 18, 519-529. [CrossRef]

25. Wagner, S.; Ribich, S.; Arrojo, R.; Castillo, M. The chemical chaperones tauroursodeoxycholic and 4-phenylbutyric acid accelerate thyroid hormone activation and energy expenditure. FEBS Lett. 2012, 585, 539-544. [CrossRef]

26. Contreras, C.; González-García, I.; Seoane-Collazo, P.; Martínez-Sánchez, N.; Liñares-Pose, L.; Rial-Pensado, E.; Fernø, J.; Tena-Sempere, M.; Casals, N.; Diéguez, C.; et al. Reduction of hypothalamic endoplasmic reticulum stress activates browning of white fat and ameliorates obesity. Diabetes 2017, 66, 87-99. [CrossRef]

27. Özcan, U.; Yilmaz, E.; Özcan, L.; Furuhashi, M.; Vaillancourt, E.; Smith, R.O.; Görgün, C.Z.; Hotamisligil, G.S. Chemical Chaperones Reduce ER stress and restore glucoes homeostasis in a mouse model of type 2 diabetes. Science 2006, 313, 1137-1140. [CrossRef]

28. Gehart, H.; Kumpf, S.; Ittner, A.; Ricci, R. MAPK signalling in cellular metabolism: Stress or wellness? EMBO Rep. 2010, 11, 834-840. [CrossRef]

29. Burgermeister, E.; Seger, R. MAPK kinases as nucleo-cytoplasmic shuttles for PPAR $\gamma$. Cell Cycle 2007, 6, 1539-1548. [CrossRef]

30. Sakamoto, T.; Takahashi, N.; Sawaragi, Y.; Naknukool, S.; Yu, R.; Goto, T.; Kawada, T. Inflammation induced by RAW macrophages suppresses UCP1 mRNA induction via ERK activation in 10T1/2 adipocytes. AJP Cell Physiol. 2013, 304, C729-C738. [CrossRef]

31. Mukherjee, J.; Baranwal, A.N.; Schade, K. Classification of Therapeutic and Experimental Drugs for Brown Adipose Tissue Activation: Potential Treatment Strategies for Diabetes and Obesity. Curr. Diabetes Rev. 2016, 12, 414-428. [CrossRef]

32. Salomone, S. Pleiotropic effects of glitazones: A double edge sword? Front. Pharmacol. 2011. [CrossRef]

33. Digby, J.E.; Montague, C.T.; Sewter, C.P.; Sanders, L.; Wilkison, W.O.; O'Rahilly, S.; Prins, J.B. Thiazolidinedione exposure increases the expression of uncoupling protein 1 in cultured human preadipocytes. Diabetes 1998, 47, 138-141. [CrossRef]

34. Asano, H.; Kanamori, Y.; Higurashi, S.; Nara, T.; Kato, K.; Matsui, T.; Funaba, M. Induction of Beige-Like Adipocytes in 3T3-L1 Cells. J. Vet. Med. Sci. 2014, 76, 57-64. [CrossRef]

35. Miller, C.N.; Yang, J.Y.; England, E.; Yin, A.; Baile, C.A.; Rayalam, S. Isoproterenol increases uncoupling, glycolysis, and markers of beiging in mature 3T3-L1 adipocytes. PLOS ONE 2015, 10, 1-14. [CrossRef]

36. Zhuo, X.Z.; Wu, Y.; Ni, Y.J.; Liu, J.H.; Gong, M.; Wang, X.H.; Wei, F.; Wang, T.Z.; Yuan, Z.; Ma, A.Q.; et al. Isoproterenol instigates cardiomyocyte apoptosis and heart failure via AMPK inactivation-mediated endoplasmic reticulum stress. Apoptosis 2013, 18, 800-810. [CrossRef] 
37. Soccio, R.E.; Chen, E.R.; Lazar, M.A. Thiazolidinediones and the promise of insulin sensitization in type 2 diabetes. Cell MeTable 2014, 20, 573-591. [CrossRef]

38. Chen, H.Y.; Liu, Q.; Salter, A.M.; Lomax, M.A. Synergism between cAMP and PPAR $\gamma$ signalling in the initiation of UCP1 gene expression in HIB1B brown adipocytes. PPAR Res. 2013, 2013. [CrossRef]

39. Xue, B.; Coulter, A.; Rim, J.S.; Koza, R. a; Kozak, L.P. Transcriptional Synergy and the Regulation of Ucp1 during Brown Adipcytes Induction in White Fat Depots. Mol. Cell. Biol. 2005, 25, 8311-8322. [CrossRef]

40. Urano, F.; Wang, X.-Z.; Bertolotti, A.; Zhang, Y.; Chung, P.; Harding, H.P.; Ron, D. Coupling of Stress in the Endoplasmic Reticulum to Activation of JNK Protein Kinases by Transmembrane Protein Kinase IRE1. Science 2000, 287, 664-666. [CrossRef]

41. Hauser, S.; Adelmant, G.; Sarraf, P.; Wright, H.M.; Mueller, E.; Spiegelman, B.M. Degradation of the peroxisome proliferator-activated receptor $\gamma$ is linked to ligand-dependent activation. J. Biol. Chem. 2000, 275, 18527-18533. [CrossRef]

42. Camp, H.S.; Tafuri, S.R.; Leff, T.; Biology, C.; Biology, M. c-Jun N-Terminal Kinase Phosphorylates Peroxisome Proliferator-Activated Receptor- gamma 1 and Negatively Regulates Its Transcriptional Activity. Endocrinology 1999, 140, 392-397. [CrossRef]

43. Molton, S.A.; Todd, D.E.; Cook, S.J. Selective activation of the c-Jun N-terminal kinase (JNK) pathway fails to elicit Bax activation or apoptosis unless the phosphoinositide $3^{\prime}$-kinase (PI3K) pathway is inhibited. Oncogene 2003, 22, 4690-4701. [CrossRef]

44. Kim, B.J.; Ryu, S.W.; Song, B.J. JNK- and p38 kinase-mediated phosphorylation of Bax leads to its activation and mitochondrial translocation and to apoptosis of human hepatoma HepG2 cells. J. Biol. Chem. 2006, 281, 21256-21265. [CrossRef]

45. Zinszner, H.; Kuroda, M.; Wang, X.Z.; Batchvarova, N.; Lightfoot, R.T.; Remotti, H.; Stevens, J.L.; Ron, D. $\mathrm{CHOP}$ is implicated in programmed cell death in response to impaired function of the endoplasmic reticulum. Genes Dev. 1998, 12, 982-995. [CrossRef]

46. Guilherme, A.; Tesz, G.J.; Guntur, K.V.P.; Czech, M.P. Tumor necrosis factor- $\alpha$ induces caspase-mediated cleavage of peroxisome proliferator-activated receptor $\gamma$ in adipocytes. J. Biol. Chem. 2009, 284, 17082-17091. [CrossRef]

47. Zha, B.S.; Zhou, H. ER stress and lipid metabolism in adipocytes. Biochem. Res. Int. 2012, 2012. [CrossRef]

48. Lockhart, D.J.; Travers, K.J.; Walter, P.; Patil, C.K.; Wodicka, L.; Weissman, J.S.; Lockhart, D.J.; Weissman, J.S.; Walter, P. Functional and genomic analyses reveal an essential coordination between the unfolded protein response and ER-associated degradation. Cell 2000, 101, 249-258. [CrossRef]

49. Ohno, H.; Shinoda, K.; Spiegelman, B.M.; Kajimura, S. PPAR $\gamma$ agonists induce a white-to-brown fat conversion through stabilization of PRDM16 protein. Cell MeTable 2012, 15, 395-404. [CrossRef]

50. Yuliana, A.; Jheng, H.-F.; Kawarasaki, S.; Nomura, W.; Takahashi, H.; Ara, T.; Kawada, T.; Goto, T. $\beta$-adrenergic Receptor Stimulation Revealed a Novel Regulatory Pathway via Suppressing Histone Deacetylase 3 to Induce Uncoupling Protein 1 Expression in Mice Beige Adipocyte. Int. J. Mol. Sci. 2018, 19, 2436. [CrossRef]

(C) 2019 by the authors. Licensee MDPI, Basel, Switzerland. This article is an open access article distributed under the terms and conditions of the Creative Commons Attribution (CC BY) license (http://creativecommons.org/licenses/by/4.0/). 